\title{
Symposium on anticoagulant therapy
}

The management of intravascular thrombosis, particularly that associated with myocardial ischaemia, is a certain source of contention: from the clinical viewpoint there is debate about the criteria upon which anticoagulant therapy is based, especially when it is intended as prophylaxis for heart disease. Even those who select this treatment fail to agree upon its duration; the role of heparin is not fully accepted; and the choice of an oral anticoagulant tends to be empirical and is frequently haphazard.

In the laboratory, the arguments in favour of using the Quick single-stage technique must be balanced against the speed and compactness of the Thrombotest and the undoubted reproducibility of the $\mathrm{P}$ and $\mathrm{P}$ test.

There does, however, appear to be some glimmer of general agreement that immediate anticoagulant therapy is indicated for the acute myocardial infarction, in which state it seems to lower mortality largely, if not entirely, by reducing the incidence and severity of thrombo-embolic complications; for cerebral thrombo-embolism; for spontaneous and post-operative deep vein thrombosis and pulmonary embolism; and after arterial disobliteration. On the other hand, there is a growing awareness that such therapy should be avoided in treating superficial thrombosis associated with venous varicosity and in all non-embolic cerebral catastrophes.

In the field of prevention, the embolic sequelae of rheumatic heart disease can largely be avoided. Many thoracic and vascular surgeons have reduced their post-operative morbidity by pre-operative prophylaxis. The prognosis in some forms of vertebro-basilar insufficiency, retinal vascular disease, Buerger's disease, and, perhaps, polycythaemia vera, can be improved. In accident and orthopaedic surgery, immediate anticoagulant treatment may considerably reduce the danger of pulmonary embolism. Some cardiological procedures, especially the pre-operative investigation preparatory to mitral valvotomy, can be freed from the risk of thrombo-embolism.

The chief bone of contention is, however, the long-term prophylaxis of myocardial ischaemia. Very simply, the two schools of thought comprise those who believe that oral anticoagulants can prevent or modify reinfarction, and those who do not. They have yet to meet in open contest, though there have been considerable rumblings. Tentative long-range literary exchanges, utilizing statistical ammunition, have inflicted a few casualties, but there is now a sinister new weapon appearing in the form of the symposium. Two international symposia have recently been held and were attended by physicians and surgeons known to favour long-term therapy, and abetted, willy-nilly, by pathologists committed to its supervision whether they favour it or not.

The verbatim proceedings of the second conference, held in London at the house of the Royal Society of Medicine, have been abstracted and are presented here without further annotation, for they contain much of the current debate. Those attending, although sometimes critical and not a little apprehensive, were all actively engaged in some form or other of anticoagulant therapy. Discussion was lively, but no doubt lacked the stimulus of antagonistic criticism. There was a definite balance of opinion in favour of prolonged prophylaxis in heart disease and this was supported by impressive and well-documented large series with control figures for comparison from Great Britain and from Norway and South Africa.

\section{THE ACTION OF ORAL ANTICOAGULANTS}

Sir George Pickering (Oxford) took the chair at the opening session, at which the first paper by R. B. Hunter (Dundee) reviewed the action of oral anticoagulants on the blood coagulation mechanism from their discovery to the present day. Oral anticoagulants, influencing both intrinsic and extrinsic coagulation systems, affected prothrombin and factors VII, IX, and X; this was complicated by a parallel alteration of thromboplastin generation.
Regarding the problem of haemorrhage, it was reasonable to say that controlled anticoagulant therapy is a safe procedure offering a risk which is certainly less than that from recurrent thromboembolism. In the question of the best laboratory control technique, Owren's recently introduced Thrombotest was favoured by Professor Hunter, who agreed with the point of view, stressed by later speakers, that there might be an unrecognized moiety occasionally affected by anticoagulant therapy, possibly a key factor in the development of haemorr- 
hagic complications, yet measured by none of the tests to be discussed at the symposium. He reviewed the present knowledge of the therapeutic effect of anticoagulants and the significance of deviations in the intrinsic and extrinsic clotting systems.

In the subsequent discussion, L. Poller (Manchester) drew attention to a depression of thromboplastin generation during phenindione therapy as demonstrated by thromboplastin generation tests routinely performed in parallel with the usual Quick single-stage control. It had been noted that the fluctuation of thromboplastin generation in a high proportion of patients failed to parallel the observed depression of the Quick prothrombin activity; this divergence might be of importance in relation to control and dosage. In three cases with spontaneous haemorrhage, thromboplastin generation was severely impaired, yet in many cases with equally severe depression there was no bleeding: this suggested that anticoagulant therapy interferes with some mechanism, other than those at present acknowledged, which might govern the incidence of haemorrhagic crises.

\section{ANTICOAGULANTS AND THE LIVER}

Sheila Sherlock (London) discussed anticoagulants and the liver, and drew attention to the fact that there is a much lower incidence of coronary thrombosis amongst patients with cirrhosis; this may be because liver disease leads to a deficiency of factors V, VII, IX, and X, coupled with a depression of lipoprotein lipase and, in many cases, there may be a platelet deficiency as well. Studies on patients with liver disease showed that oral anticoagulants had no effect on liver function tests but, because of the apparent reduction in clotting in such patients, anticoagulant therapy is seldom necessary. Phenindione tolerance tests were performed on patients with well-compensated liver disease, using first $2 \mathrm{mg}$. and then $4 \mathrm{mg}$. $/ \mathrm{kg}$. body weight in a single dose: the single-stage prothrombin index rose higher and remained elevated longer in these patients compared with subjects without liver disease. Jaundice due to phenindione sensitivity is extremely rare if, indeed, it has ever been correctly reported. In the cases described, it was not possible to assess the particular type of jaundice produced but the pattern of toxicity might be similar to what one would expect with drugs containing a benzine ring.

In the subsequent discussion, it transpired that no member of the symposium had experience of jaundice as a result of anticoagulant therapy nor had any speaker personal knowledge of a case.

H. W. Fullerton (Aberdeen) referred to studies of the recalcified plasma clotting time after a high fat intake. He found that the usual acceleration \&f this time after three-and-a-half hours failed to occi in patients on phenindione therapy either for recent infarction or on a long-term basis for myocardia ischaemia. A. S. Douglas (Glasgow), commenting of the lower incidence of atheroma in cirrhotics an the coincidental increase of fibrinolytic activite, recognized this to be a feature of importance assessing prothrombin times in patients with live्d disease. He suggested that the fibrinogen level should be studied because of the possibility of intense fibrinolysis. In contrast antihaemophilic globulin extraordinarily stable in such patients, although th factor is also susceptible to proteolytic degradatio $\vec{m}$

R. G. Macfarlane (Oxford), speculating on the coincidental depression by phenindione of for apparently dissimilar clotting factors, raised the possibility of a common carrier protein of similar physical properties. He wondered whether anticoagulant drugs might inhibit other forms of proter synthesis, perhaps carried out in the liver. F. Kolle (Zurich) remarked on an increase in heparin anti. thrombin activity in addition to increased fibrin lysis in advanced cirrhosis.

\section{LABORATORY CONTROL OF ANTICOAGULANT THERAPY}

There followed two sessions, under the chairma ship of R. G. Macfarlane, dealing with aspects laboratory control. P. de Nicola (Pavia) outlined the place of the Quick single-stage test, re-evaluatin it on the basis of newly discovered factors. The problems concerned with the choice of thromber plastin were mentioned and general directions wefe given for ensuring safe anticoagulant contro․․ P. A. Owren (Oslo), discussing the laboratory control of anticoagulant therapy, referred to the well-known shortcomings of the Quick single-stage test: that it is too sensitive to variation of factor $\nabla$ and fibrinogen; that the specified calcium concentre tion becomes susoptimal in the haematocrit if the tested blood is outside the normal range; and tha Quick's original method with rabbit brain thrombo plastin gives insufficient sensitivity, because of th short range of only seven seconds from the controp to the therapeutic level, when using an adsorbed plasma dilution curve. He went on to discuss how these shortcomings had been overcome first by the $\mathbf{P}$ and $\mathbf{P}$ test and, more recently, by his Thromb $\overrightarrow{\sigma_{-}}$ test. The latter was designed to be extremely simple to use and it takes into account depression of prothrombin and factors VII, IX, and X, at the sam? time providing a suitable reagent for universal use. He stressed that results should always be given as percentage of normal by the use of a correlatiog 
curve. This should be prepared by serial dilutions of a normal standard plasma in adsorbed normal plasma. The widely adopted practice of diluting normal plasma with saline or buffer results in false prolongation of prothrombin times at higher dilutions, because of alteration of fibrinogen and factor V concentration. Further, neglecting a constant citrate concentration in serial dilutions increases the error. In his opinion, the use of the Thrombotest permitted large-scale anticoagulant therapy control without these drawbacks and without the unnecessary introduction of error into laboratory procedures. He speculated upon the fact that it may be depression of factor IX which is most closely associated with the tendency of a given patient to show haemorrhagic manifestations. In discussing the arbitrary selection of a therapeutic range, Professor Owren referred to a percentage activity based on dilution curves, with an adsorbed plasma diluent. He selected a $10-25 \%$ therapeutic range: below $10 \%$, haemostasis became defective, presumably largely due to factor IX depression, and above $25 \%$, he felt that the therapeutic results were not good enough to justify long-term therapy. With the Thrombotest he was maintaining patients at a level much nearer to $10 \%$ with perfect safety. $\mathrm{He}$ provided figures to show that, in the treatment of venous thrombosis, a level of $15 \%$ is highly efficient in preventing further thrombo-embolism in, for example, rheumatic heart disease or post-operative states. As regards arterial disease, he was less confident, but showed results that indicated a highly significant improvement in the re-infarction rate when comparing groups of patients treated within a range below $20 \%$ with those maintained at $50 \%$ activity. He stressed the importance of maintaining therapy at the maximum intensity consistent with safety. W. Walker (Dundee) then described his experience with Thrombotest, pointing out that recent work had been free of the difficulties earlier encountered with both capillary and venous blood. These difficulties are of the kind that might initially discourage a routine laboratory but can be avoided by observing simple precautions in technique; illustrating this, he outlined the technique in detail and demonstrated advantages of the method, although the testing of venous blood stored overnight is not so straightforward.

T. B. Counihan (Dublin) opened the subsequent discussion and drew attention to difficulties experienced in Eire because so much treatment is based on postal samples. G. A. McDonald (Aberdeen) enquired as to the best site for removing the capillary specimen required for the Thrombotest, and it was generally agreed that the side of the pulp of the finger or the lobe of the ear were best incised by a narrow scalpel blade. One of the drawbacks of Thrombotest, when used as a capillary assessment, is the need for a relatively large amount of capillary blood, and many patients prefer the use of an intravenous needle.

There was some general discussion on the question of a rebound phenomenon following the discontinuance of anticoagulant therapy. No statistical evidence is available to assess this, but there was a general feeling that such a possibility could not be ignored and that anticoagulant therapy should be 'tailed off' in order to cover the possibility of an increased tendency to clot. No suggestion as to how long this might be required was forthcoming. Owren's figures showed that most patients died in the second or third month after discontinuance of therapy and this appeared to be too long a latent period for the rebound phenomenon to be implicated. Experience in Norway showed that the tendency increased with age but that it lasted only a few days.

Rosemary Biggs (Oxford) presented a paper on the laboratory control of anticoagulant therapy, in which she reviewed experience up to date. She drew attention to the fact that for people who were interested in the study of coagulation mechanisms the problem of control presented little difficulty, but where tests were widely employed in routine use by people who had no especial interest, then random variability occurred. She considered the Quick single-stage test to offer a high degree of random variability. She considered Thrombotest probably to be the method of choice for smaller laboratories without the services of individual experts. Her experience with the $\mathbf{P}$ and $\mathbf{P}$ test led her to believe this to be the best available method of assessment in larger laboratories, for there is much less random fluctuation and a more obvious trend in relation to dose and a greater sensitivity in the near normal range. As this test involves two reagents instead of one, which have to be standardized, it is only reliable when used in laboratories undertaking coagulation studies on a substantial scale.

\section{ANTICOAGULANT TREATMENT AND THE PATHOLOGIST}

R. Aidin (Worthing) followed with a paper discussing the position of treatment from the point of view of the pathologist and there followed a lively discussion on the comparative merits of Thrombotest and the Quick single-stage method. Dr. Biggs concluded the discussion with the comment that it was probably better for those who have no primary interest in coagulation mechanisms to use a method already shown to be feasible by the wealth of other 
people's experience rather than trying to pit their own lack of experience against insuperable odds.

The last session of the first day, under the chairmanship of H. W. Fullerton, started with a paper by $F$. Koller (Zurich) discussing his experience with the use of fibrinolysins in the treatment of thromboembolic disease. He reviewed briefly the mechanism of the fibrinolytic system and contrasted it with that of intravascular coagulation. Although antagonistic, these two biological phenomena possess many similar features. Both are dependent upon the final production of a proteolytic enzyme, thrombin or plasmin. In each system the proteolytic enzyme is derived from an inactive plasma precursor, the activation of which can be produced in both systems by two different mechanisms: by a tissue activator or a plasma activator, the latter being generated exclusively by constituents of the blood itself.

Clinical experience was based on the intravenous use of streptokinase as an activator of mechanisms themselves activating plasminogen within the vascular system. An alternative method, by the intravenous infusion of human plasmin itself, probably acts by a similar route because plasmin preparations currently available are contaminated by the streptokinase used in their preparation and it is likely that any therapeutic efficiency is directly proportional to the degree of contamination present. Professor Koller presented a preliminary report on the results of treatment of 103 patients receiving parenteral fibrinolytic activators. Purified streptokinase, plasmin, Pyrexal (a lipopolysaccharide of bacterial origin), nicotinic acid, and Complenin (a combination of nicotinic acid) were used singly. The dose of each preparation was estimated arbitrarily for each patient by assessing the amount of the preparation required to produce dissolution of a clot within 10 minutes: by thromboelastograph methods, considerable individual variation was experienced, but it was found possible to plan a general dose schedule for each patient, who usually received an intravenous infusion over one or two hours. Plasminogen is rapidly consumed and two hours appeared to be an optimal time for maximal activation. Beyond this, further streptokinase has no plasma substrate to act upon but, as plasminogen can be adsorbed on to the surface of the fibrin, streptokinase may still continue to have an effect after plasminogen has disappeared from the plasma. Clinical experience shows that infusions can be continued for $\mathbf{2 0}$ or $\mathbf{3 0}$ hours. Venous thrombosis of the leg and pulmonary embolism were treated with apparent success, but in arterial thrombosis results were equivocal, for distal infarction areas remained necrotic, irrespective of any clearance of the blocked artery.
Side-effects of this treatment, such as rigors, were not seen when highly purified preparations of: streptokinase or plasmin were used. Haemorrhage was prone to occur with the use of higher doses because fibrinogen, factor V, and factor VIII weres: attacked, in addition to the pre-formed fibrin. The concentration of these factors is not reduced below $10 \%$ to $30 \%$, however, and any bleeding tendenow is usually mild. Tests in vitro to estimate clotting ma $\vec{\phi}$ be vitiated and given a spuriously lengthened time because the breakdown products of fibrinolysis mage interfere with the further polymerization of fibrits Allergic reactions were noted only with less purifiel preparations and well controlled fibrinolytic treats ment was found to produce less haemorrhage than, for instance, heparin therapy.

The safety of intravenous fibrinolysin therapy cag be enhanced by the availability of a fibrinolysin antagonist: $\Sigma$ amino-caproic-acid has been proved to be the most efficient of these agents and is very closely related to the essential amino-acid lysine? It is harmless and can be given in doses of 10 or even $30 \mathrm{~g}$. per day by mouth or a slightly lower dose intravenously. It is well tolerated and, although rarely required in fibrinolytic treatment, it imme diately corrects any spontaneous bleeding.

\section{SELECTION OF PATIENTS FOR LONG-TERM ANTICOAGULANT TREATMENT}

P. A. Owren (Oslo), in a second paper, discussea the selection of patients for lifelong anticoagulan therapy and described Norway's experience of the organization of large groups of patients. Bearing mind that anticoagulant therapy is prophylactic rather than curative, he emphasized the value of lifelong therapy in any clinical disease having a high tendency for venous or arterial thrombo-embolig complications. The importance of starting this before the disease is too far advanced was stressed; in an clinical studies so far published the inclusion advanced cases has largely masked the results of therapy for the rest of the group.

The general structure of a convenient andb economical organization is dependent primarily of selection into groups of varying priority. Patienfs in Norway have been classified into two main groups: a high priority group of patients on whol anticoagulants have a marked effect on mortalito and disablement, and a group with second priority where the beneficial effects of anticoagulant theraps are less well established. The high priority group can be divided into five classes. First rheumatic hea disease with embolism; without therapy the fre quency of recurrent embolism is high but the effece of therapy is dramatic and results so far raise the 
question of whether prophylaxis might start before the first embolic episode, since this is often catastrophic. The frequency of embolism in unselected patients with rheumatic heart disease is $20 \%-30 \%$ over a 10-year period; in a group of 30 patients receiving long-term therapy none has had an embolism over a period of three years. The second class comprises those suffering from angina pectoris of less than two years' duration; here also anticoagulants have had a marked effect. Of 470 receiving long-term therapy, the mortality was only $3.6 \%$ per year against the usual mortality in similar patients not treated of $6 \%$ to $10 \%$. The third class are patients with one attack of myocardial infarction and, in this large group, patients were selected who had had no angina or had had the condition for less than two years. Results showed that new infarctions were more frequent in patients with more than one infarction before therapy, both in the treated groups and in the control groups: it is safe to assume that anticoagulant therapy is most effective at an early stage of the disease but it does not appear to protect this class of patients to any appreciable extent.

The fourth high priority class in those suffering from atherosclerosis of the legs without signs or symptoms of coronary or cerebral arteriosclerosis; a high proportion of these ultimately died because of coronary disease or some cerebral catastrophe. The mortality rate of 140 such patients treated for four years was $2.7 \%$ to $3.7 \%$ per year. The fifth and final class receiving high priority suffer intermittent insufficiency of the cerebral circulation, presumably due to thrombosis. Experience in Norway is limited but strongly suggests that these patients should have anticoagulant therapy, a point which did not receive support from subsequent speakers.

The second main group of patients receiving less priority can be subdivided into three main classes: 1, rheumatic heart disease without embolism; 2, angina pectoris of more than two years' duration; and 3, two or more myocardial infarcts. For these classes there is only suggestive evidence that anticoagulant therapy decreases mortality and it does not appear justifiable to insist on treatment.

Professor Owren believed that in Norway probably $1 \%$ of the population in the next 10 years will require long-term anticoagulant therapy; he next discussed the problem of handling such a large number of patients using Thrombotest as a measure of control and, finally, indicated that this organization is inexpensive and that the laboratory costs amounted to about $£ 1$ per year per patient.

In opening the discussion which followed, M. F. Oliver (Edinburgh) provided figures derived from an analysis of patients discharged from the Edinburgh Royal Infirmary during 1959 following treatment of angina, acute coronary insufficiency, and acute myocardial infarction. From these figures he calculated that for the country as a whole 46,000 patients will start anticoagulant treatment every year. The approximate cost for this number of patients would be $£ 850,000$ a year and, if they are going to have lifelong anticoagulant therapy, allowing for the mortality known to occur in people who do not receive it, then some $£ 6 \mathrm{~m}$. will be spent every year in giving this form of treatment for coronary disease. By using Professor Owren's selected categories of priority it should be possible somewhat to reduce the cost.

A second point introduced by Dr. Oliver was consideration of the comparatively little protection given to women as opposed to men. Combining the results of the various large-scale trials, it would seem that there is a case for expanding investigations to find out whether women enjoy the same degree of protection as men. In his opinion they do not and, by confining therapy not only to males the number of people involved could be further reduced. By making use of every possible reduction, it would seem that some 13,500 patients would still require attention, and, if they attended every three weeks, this means 4,500 attending hospitals and clinics in Britain each week. The cost of such a service would be considerably in excess of that already allocated for the entire poliomyelitis immunization programme.

Professor Owren (Oslo) drew the attention of the symposium to the tendency to discuss the cost without bearing in mind the parallel considerable saving in both time and money effected by anticoagulant therapy. For example, admissions to hospital could be reduced and patients could return to work much sooner.

\section{ACUTE CORONARY INSUFFICIENCY}

On the second day, under the chairmanship of Professor O. Meyer (Wisconsin), P. H. Wood (London) opened with a paper on acute coronary insufficiency. He first defined the condition as an unstable state in which the coronary circulation is insufficient to meet the full metabolic demands in the myocardium under resting conditions but is sufficient to prevent necrosis. There is a background of ischaemic heart disease in at least $80 \%$ of cases; the onset is sudden and the patient may become totally incapacitated within 24 hours, still without cardiac infarction. The natural history of the condition shows that cardiac infarction develops in approximately $22 \%$ within a period of two months. Indeed this same state precedes about a quarter to 
one-third of all cardiac infarctive episodes. The overall mortality is about $25 \%$. Necessarily there are no laboratory data indicating cardiac infarction; the E.S.R. is normal, the temperature is normal, and the serum transaminase and lactic dehydrogenase activity is also normal. One hundred and fifty consecutive cases of subacute coronary insufficiency were studied, 100 being treated with anticoagulants and the other $\mathbf{5 0}$ serving as controls. When the effect of treatment became appreciable, it was no longer considered justifiable to continue a control group and all patients received therapy. The rate of infarction occurring within two months of seeing the patient was $3 \%$ among those treated and $22 \%$ among those untreated. The minimum mortality rate, allowing for untraced cases, was $6 \%$ in the treated group and $30 \%$ in the untreated controls. As far as their final state of health was concerned, the difference was less noticeable, the treated patients only doing twice as well as the controls. A year or two later these figures showed a clear indication that anticoagulant therapy must be given when a patient has acute coronary insufficiency thought to be due to thrombosis. Treatment should start with heparin and phenindione given simultaneously.

\section{EVALUATION OF LONG-TERM ANTICOAGULANT TREATMENT}

A. S. Douglas (Glasgow) dealt with the results of the Medical Research Council's trial on long-term therapy. The trial had now been completed but it was permissible for him to speak only of results from the time of the first entry of cases to the trial in November 1955, up to the time of the initial publication of the report in March 1958. He commented upon some of the criticisms which had been directed at this trial and enlarged on some of the difficulties which had been encountered. As regards treatment of women, numbers were too small in the M.R.C. trial to gain adequate evidence, but by assessing the three trials which have had a similar design there had been no demonstrable difference in mortality in females and only a borderline improvement in the rate of infarction. Dr. Douglas had also calculated the requirements of long-term anticoagulant therapy: the incidence of patients who have suffered an infarction within the last two years and are still alive is 1 in 380 and the incidence of patients suffering from angina is 1 in 170; these figures are based on a survey of an area of west Scotland covering a population of 70,000 , and the overall incidence of patients in both these categories approximated 1 per 100 .

M. M. Suzman (South Africa) presented a paper on the evaluation of the effect of long-term anti- coagulant therapy on the prognosis of coronary artery disease. This had involved a considerable: amount of work and included documented observavis tions on more than 2,000 patients. It was shown that mortality rates in survivors of myocardial infarctior maintained on long-term treatment are consistentlye lower than the rates observed in survivors whosed treatment with anticoagulants was limited to the acute phase, and this difference in death rate was observed in all age groups. More than 3,000 patient $\vec{\rho}$ in the long-term group of treated cases were com $=$ pared with 4,800 in the entire short-term group, the five year survival rate being a little over $50 \%$ in the untreated group and about $75 \%$ in the treated group: A history of infarction before the presenting attack materially increased the mortality in both groups of patients but to a far greater degree in those noe, receiving long-term anticoagulants. Amongst patients who discontinued long-term treatment, the subsequent mortality rates were higher than in those whos maintained treatment without interruption.

In the subsequent discussion C. S. McKendrick (Liverpool) and K. Ball (London) referred to the problem of handling patients in large numbers, and their remarks were echoed by many other speakers Dr. Ball referred to the observations in the M.R.C study that both deaths and recurrence effects were most apparent in the first three months; as a resule he had adopted the practical compromise of placing patients suffering from their first infarct on anti은 coagulants for three months after they leave hospita市 (a total of four months altogether) and then tailing off therapy over the next six weeks.

A. Morgan Jones (Manchester) criticized the use. of the term 'acute coronary insufficiency' as bein $\overline{\mathscr{E}}$ neither a pathological entity, such as thrombosis: nor a symptom, such as angina. He recalled thae ischaemic heart disease is known to be a phasie disease with periods of activity and periods of quiescence, the former lasting anything up tog 18 months and the latter 10 or more years. Hes suggested that cases should be differentiated and․ that anticoagulants in the first instance should be limited to those known to be suffering from the active phase of the disease. He speculated that the better results shown by Dr. Paul Wood's study were్ partly due to the fact that all his patients by definitionwere in the active phase; the poorer results of the longer M.R.C. trial might be due to the fact thaf only a small proportion of patients after a myo cardial infarct remain in the active phase. S. Sevitio (Birmingham) felt that there could be no doub $\overrightarrow{0}$

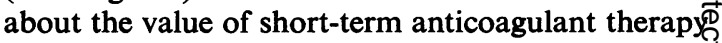
in myocardial infarction. He stressed that the beneficial effects were not necessarily related to the underlying cardiac condition but a high proportion 
of patients were saved who would otherwise have died, because venous thrombosis and pulmonary embolism had been prevented. In this respect it was important to know what the incidence was of recent intravascular coronary thrombosis amongst those who died in a treated series compared with the incidence in those who died without anticoagulant treatment.

R. L. Richards (Glasgow) described the indications for anticoagulant therapy in peripheral arterial disease, emphasizing that clinical success can be gauged only by prophylaxis and not from any curative effect. Anticoagulant therapy has an important place in the management of acute and chronic arterial occlusion, especially of peripheral vessels, and as an adjunct to disobliterative surgery. J. L. Stafford (London) illustrated the experience gained in the organization of a large out-patient clinic covering long-term anticoagulant therapy in a teaching hospital. He drew attention to the hidden costs incurred by the transport of patients to and from clinics and the mounting expenditure of professional and technical time devoted in the laboratory to this commitment.

Sir W. M. Morrow (Australia) spoke on the problem of long-term anticoagulant therapy in the country districts of Australia and described the difficulties facing general practitioners having minimal laboratory facilities. D. Stark Murray (Kingston) opened the discussion by referring to his own experience in running a domiciliary service in his own region where the majority of patients were nursed at home; he described a bedside technique developed for the control of anticoagulant therapy which seemed to work satisfactorily. But he also stressed the cost of the service in both time and money and the impact of this commitment on the other services rendered by a clinical pathology laboratory.

E. Petrie (London) described the general practitioner's role in anticoagulant therapy and his own endeavours to control treatment, utilizing a modified prothrombin activity assessment which he carried out himself. S. Sevitt (Birmingham) presented a paper on anticoagulant prophylaxis against venous thrombosis and pulmonary embolism in injured patients, and drew upon his considerable experience at the Birmingham Accident Hospital. It was now their accepted practice to advocate prophylactic anticoagulant therapy for all patients who were considered to be at risk of venous thromboembolism. Indeed, it was the rule to provide this treatment, unless there was a contraindication, for all those over the age of 45 and admitted for a period of bed-rest predicted to be longer than three or four days. Figures were presented to indicate that the incidence of pulmonary embolism had been reduced by as much as $80 \%$ or $90 \%$.

\section{ANTICOAGULANT TREATMENT OF CEREBRAL VASCULAR DISEASE}

C. H. Milliken (Rochester, U.S.A.) presented evidence from the Mayo Clinic on the use of anticoagulants in cerebral vascular disease. He stressed that such treatment was strictly limited to a few categories of patient and these, although important, comprise only a small proportion of patients with cerebral disease. He eschewed long and complicated classifications and preferred to use the simple term 'stroke'. Of patients suffering a primary cerebral episode there are three main groups: 1 , Those with incipient or impending stroke in whom neurological examination is generally normal, but there may be some warning indicating that a stroke is likely; 2 , advancing stroke in patients suffering an active progression of neurological deficit, during the period of observation extending over a matter of hours; and 3 , those with a completed stroke where the neurological deficit, although of variable degree, is stable but may be followed by improvement over days or months. Of these three conditions only the first should receive anticoagulant therapy; it is convenient to make a distinction between the two vascular systems involved in this category. First, because there is a difference in mortality until ultimate thrombosis and, secondly, because there is a difference in the incidence of haemorrhage in the two systems with or without anticoagulant therapy. Thus one speaks of intermittent insufficiency in either the vertebral-basilar or the carotid systems. Selected cases with advancing thrombosis in the vertebral-basilar system might also benefit from therapy if the diagnosis is distinct. Apart from these sharply defined and strictly limited categories, only the recurrent embolic phenomena associated with cardiac pathology are suitable for anticoagulant therapy.

J. Marshall (London) also dealt with the use of anticoagulants in cerebrovascular disease, outlining the difficulties in diagnosis, especially in distinguishing haemorrhage from infarct. He considered that the indications for anticoagulant therapy at the present time are limited, first to those with acute cerebral infarction due to embolism; secondly, those with known embolic infarction if seen at a stage at which development is slow and still progressing; thirdly, in patients experiencing recurrent ischaemic episodes in the vertebro-basilar territory; and 
fourthly, in patients prone to have a partial stenosis of the internal carotid artery which is giving rise to symptoms.

The final paper by R. W. Emanuel (London) was on the treatment of pulmonary hypertension and mitral valvular disease in relation to anticoagulants. This field of clinical study had received little attention. Experience at the National Heart Hospital and Brompton Hospital was based on four classes of patient. In the treatment of mitral stenosis complicated by severe pulmonary hypertension it was found that anticoagulants protect patients from fatal thrombo-embolism while waiting for cardiac catheterization and operation. In mitral stenosis uncomplicated by pulmonary hypertension, anticoagulants appear to diminish the risk of systemic emboli. Subacute thrombo-embolic pulmonary hypertension can be cured if anticoagulants are used sufficiently early, but this form of treatment appears to have no effect on the natural history of primary pulmonary hypertension and, indeed, may cause fatal haemorrhage in cases of the Eisenmenger syndrome.

During the final session of the symposium a panel discussion was held during which time questions presented during the meeting were answered extemporaneously. Professor Sir George Pickering (Oxford) took the chair and his fellow members were Professor R. B. Hunter (Dundee), Dr. C. H. Milliken (Rochester, U.S.A.), Professor P. A. Owren (Oslo), and Dr. P. H. Wood (London).

The first question from Dr. Sevitt was, 'It is now established that effective oral anticoagulant therapy for cases clinically diagnosed as having deep vein thrombosis of the lower limb reduces the incidence of fatal pulmonary embolism from $80 \%$ to $90 \%$. Why is this so? Why is it that anticoagulant therapy seems to prevent detachment of the clot?'

Some surprise was expressed that the rate could be cut down by as much as $80 \%$ to $90 \%$. It was suggested that efficient anticoagulant therapy functioned by reducing the tendency for the friable end of the clot to break off. Secondly, the prevention of further extension of the thrombus is bound to reduce the possibility of complications. The size of the embolus is reduced; it is probably possible to reduce the mortality of pulmonary embolism by $90 \%$ but unlikely that the incidence can be reduced by such a considerable figure.

Dr. R. G. Macfarlane (Oxford) enquired, 'Should a pathologist or a clinician, or both, control anticoagulant therapy?' This question evoked a great variety of personal opinions which, on the whole, reflected the specialty of the person commenting. Generally speaking clinicians, particularly physicians, preferred to maintain their own clinical control, but accepted the necessity for laborator surveillance, and, with it, the collaboration of the clinical pathologist.

The development of this form of hospital controt in Britain has been haphazard and many people would appreciate guidance on how best to deal witks the problem. At the moment, however, man clinicians remained disinterested and it was bettery in such circumstances, for the clinical pathologist to. assume control, so long as he maintained a close liaison with both the physician and the genera practitioner.

The introduction of the Thrombotest might mak it possible for a clinician relatively inexperienced in. laboratory methods to maintain an anticoagulant clinic in the way that the hospital diabetic clinic has been organized; this possibility, with the implication that technical staff would work directly under the control of the practising physician, has yet to be. explored. There is some difference between general hospital and a teaching hospital, where highly specialized laboratory facilities may be more readily available. This question of the responsibility of clinical supervision of groups of patients raised $\&$ matter of considerable importance regarding the relationship of the clinical pathologist and his colleagues.

Professor Counihan (Dublin) asked, 'What is the size of risk of subintimal haemorrhage in coronaro atheroma when anticoagulants are begun? Is there a period of time when this risk is much greater i relation to a clinical episode of coronary occlusion?

There appeared to the Panel to be no direct answer possible, as the presumption of subintimal. haemorrhage leading to coronary occlusion has never been adequately confirmed. If it occurred ats all frequently it was felt that many would have hậ]. the experience of a patient in a state of acute coronary insufficiency developing massive occlusio under therapy, yet this in fact is very unusual. It was considered that such a risk was not serious if indee it existed.

Dr. D. G. Chalmers (Cambridge) enquired, 'Doe the Panel feel it necessary to administer phenindione in divided daily doses to patients on long-ternf therapy?' Opinion was evenly divided. From the्E scientific point of view there was no necessity to divide the daily dose so far as stability was conto cerned. Some were in favour of a single dose to minimize the patient's tendency to forget; some felep it better to divide the dose so that, in the unlikelyo event of trouble, it was easier to correct an overdose The administrative convenience of a single daily dose appeared to be of greater benefit and was adopte by most large clinics using phenindione. It was generally agreed that the use of divided daily doses 
of the long-acting coumarin derivatives is entirely unnecessary.

Dr. S. H. Davis (Edinburgh) asked, 'In view of the increasing use of long-term anticoagulant drugs, would it not be advisable for all hospitals to issue a nationally agreed standard instructional pamphlet and to incorporate in it a page for the inclusion of the type of drugs used, dosage and control data. If so, would the Panel decide the detailed contents?' After consideration and a somewhat humorous discussion, it was generally agreed by the Panel that written instructions must be given to the patient and that it is administratively desirable to provide written information on the nature of the drug, the dose, the patient's blood group, and the address and telephone number of the hospital assuming control. It was widely recognized that no two centres and no two individuals would ever agree on the wording of an instructional pamphlet and it was considered to be undesirable for any such communication to be the product of any central authority. Although it was desirable for instructions to be available, it was agreed that no pamphlet should be designed to take the place of the clinical interview between the doctor running the anticoagulant clinic and the patients coming under his supervision. On the whole, the possibility of a pamphlet proving satisfactory to all concerned appeared to be quite remote. Patients who were mentally incapable of cooperating with the written instructions were possibly better avoided in the first instance.

Professor Koller (Zurich) asked, 'How long should one continue to treat pulmonary embolism in order to avoid cor pulmonale?'

It was felt that anticoagulant therapy must be continued until the pulmonary vascular resistance is normal. In the case of a young man with an efficient dual circulatory system, this might be effected within six to 12 weeks; on the other hand, an elderly person with recurrent embolism may have to receive therapy for life and, between these two extremes individual variations were considerable. For a major pulmonary infarction nine to 12 months' therapy is probably necessary. Otherwise the patient may pass into a period of ill-health, which may last a year or more, and which is ascribed to pulmonary hypertension.

The Panel had no useful comment to offer as to whether acute coronary thrombosis occurred at any specific time of the day or week. On the question of the use of anticoagulants for cerebral embolism, there was disagreement. Some members of the Panel felt that anticoagulants, both heparin and phenindione, should be started immediately the diagnosis is made, but this might be followed by an increasing bleeding into the infarction and this danger was present for 24 to 72 hours after the initial occlusion.
With this in mind, some workers withhold anticoagulants for at least four days, giving them ultimately with the philosophy of preventing the next embolic event rather than materially changing the infarct which has already occurred. The majority of the Panel favoured abandoning the use of heparin, but one member, on the contrary, felt that any form of anticoagulant therapy was indicated immediately, because most cases of cerebral embolism involved multiple emboli; the maximum disability is reached at once and there are grounds for treatment as quickly as possible.

The next question asked the opinion of the Panel about the administration of heparin in cases of cardiac infarction while awaiting the action of oral anticoagulants. Although there is no statistical evidence one way or the other, all were agreed that heparin should be started at once and continued for at least 24 hours.

Dr. A. G. W. Whitfield (Birmingham) asked about the advantages and disadvantages of long-acting anticoagulants compared with phenindione. The Panel agreed that it is best in any given centre to use the preparation to which those in charge of therapy have become accustomed. This differed from area to area and from country to country. Phenindione is the most popular because of its rapid action and is easier to control on an outpatient basis. Thus, in Europe, the slower acting coumarin derivatives are generally used only for cases where phenindione proves unsuitable.

As outpatient therapy is likely to increase, there will be a substantial rise in the number of patients requiring an alternative form of therapy and it is advisable for clinicians to gain experience in the use of at least one preparation in addition to phenindione. Of those quoted, warfarin sodium or phenylpropylhydroxycoumarin appear to be the most popular.

On the question of switching from one preparation to another, it was difficult to be dogmatic, but it is possible to transfer from the quick-acting phenindione to the slower-acting preparation without delay and this is the course usually required; the change from a slow-acting to a swift-acting preparation runs the risk of potentiation and must be controlled carefully.

The Panel agreed that the treatment of haemorrhagic complications of anticoagulant therapy was best effected by discontinuing the drug for a day or two; scarcely ever should it be necessary to give vitamin $K_{1}$ because of the possibility of a rebound coagulative tendency. The accumulated experience of many centres has shown that haemorrhagic complications are much less important than was hitherto appreciated and that it is perfectly safe 
merely to stop the drug and wait to see what happens. The one exception seems to be haemorrhage into the eye, but such complications are extremely rare. Selection of the patients before admitting them for long-term anticoagulant therapy should reduce the possibility of dangerous bleeding.

The last question by Dr. A. G. Signy (London) was, 'Would the Panel advise in cases of sensitization to an oral anticoagulant a short period of dose reduction before changing over to a newer drug; 을 under what conditions would the Panel change the drug?' The Panel agreed that sensitization was un- $\stackrel{\text { ? }}{+}$ common and was usually shown by a rash or, occasionally, diarrhoea. Most people switched흘 directly and in comparable doses. With regard to $\frac{\bar{p}}{\partial}$ phenindione, some of the sensitivity may be $\underset{\propto}{\mathbb{D}}$ associated with the benzine ring in this prepara-i tion.

\section{Broadsheets prepared by the Association of Clinical Pathologists}

The following broadsheets (new series) are published by the Association of Clinical Pathologists. They may be obtained from Dr. R. B. H. Tierney, Pathological Laboratory, Boutport Street, Barnstaple, N. Devon, $\frac{3}{\mathrm{~N}}$ price 1s. each.

1 The Disc Technique for the Rapid Determination of Bacterial Sensitivity to Antibiotics. 1952. R. W. FAIRBROTHER.

2 Determination of Sensitivity of $M$. tuberculosis to Streptomycin. 1952. R. L. vollum.

3 The Detection of Barbiturates in Blood, Cerebrospinal Fluid, Urine, and Stomach Contents. 1953. L. C. NICKOLLS.

4 The Estimation of Carbon Monoxide in Blood. 1953. D. A. STANLEY.

5 The Identification of Reducing Substances in Urine by Partition Chromatography on Paper. 1953. G. B. MANNING.

6 The Paul-Bunnell Test. 1954. R. H. A. SWAIN.

7 The Papanicolaou Technique for the Detection of Malignant Cells in Sputum. 1955. F. HAMPSON.

8 Investigation of Haemorrhagic States, with Special Reference to Defects of Coagulation of the Blood. 1955. E. K. BLACKBURN.

9 Daily Fat Balance. 1956. A. C. FRAZER.

10 Mycological Techniques: (1) Collection of Specimens. 1956. R. W. RIDDELL.

11 Mycological Techniques: (2) Cultural Isolation. 1956. R. W. RIDDELL.

12 Techniques for Demonstrating L.E. Cells. 1956. J. V. DACIE and L. S. SACKER.

13 The Identification of Serotypes of Escherichia coli Associated with Infantile Gastro-enteritis. 1956. JOAN TAYLOR.
14 The Determination of Serum Iron and Serum $\vec{\odot}$ Unsaturated Iron-binding Capacity. 1956.ARTHUR JORDAN.

15 The Estimation of Faecal 'Urobilinogen'. 1957. C. H. GRAY.

16 Preservation of Pathological Museum Speci-⿳亠丷厂巾 mens. 1957. L. W. PROGER.

17 Cultural Diagnosis of Whooping-cough. 1957. $\overrightarrow{\overrightarrow{0}}$ B. W. LACEY.

18 The Rose-Waaler Test. 1957. c. L GREENBURY.

19 The Laboratory Diagnosis of Fibrinogen De-o응 ficiency. 1958. R. M. HARDISTY.

20 Investigation of Porphyrin/Porphyria. 1958. C. RIMINGTON.

21 Quantitative Determination of Porphobilinogen and Porphyrins in Urine and Faeces. 1958. c. RIMINGTON.

22 Investigation of Haemolytic Anaemia. 1959. J. G. SELWYN.

23 The Dried Disc Technique for Bacterialn Sensitivity Tests. 1959. R. W. FAIRBROTHERN and J. C. SHERRIS.

24 Safe Handling of Radioactive Tissues in the Laboratory and Post-mortem Room. 1959. R. C. CURRAN.

25 Titration of Antistreptolysin O. 1959. H.O GOODER and R. E. O. WILLIAMS.

26 The Periodic Acid-Schiff Reaction. 1959. A. G. E. PEARSE.

27 The Laboratory Detection of Abnormal Hae- $\frac{2}{2}$ moglobins. 1960 . H. LEHMANN. 